\title{
Emergency Repair of a Perforated Mycotic Aneurysm with a Self-made Pericardial Tube-graft
}

\author{
Nawras Diab ${ }^{1,2}$ Clarence Pingpoh ${ }^{1,2}$ Matthias Siepe ${ }^{1,2}$ Friedhelm Beyersdorf ${ }^{1,2}$ \\ Ahmed Kharabish ${ }^{3,4}$ Martin Czerny ${ }^{1,2}$
}

${ }^{1}$ Department of Cardiovascular Surgery, University Heart Center

Freiburg Bad Krozingen, Germany

2 Faculty of Medicine, University of Freiburg, Germany

${ }^{3}$ Department of Radiology, University Heart Center Freiburg, Germany

${ }^{4}$ Radiology Department, Cairo University, Egypt

Thorac Cardiovasc Surg Rep 2018;7:e9-e11.

\begin{abstract}
Address for correspondence Nawras Diab, MD, Department of Cardiovascular Surgery, Universitats-Herzzentrum Freiburg Bad Krozingen GmbH, Suedring 15, Bad Krozingen 79189, Germany (e-mail: nawras.diab@universitaets-herzzentrum.de).
\end{abstract}

\begin{abstract}
Keywords

- aneurysm

- cardiovascular surgery

- thoracic surgery

A 63-year-old female with a history of kidney transplantation was admitted for emergency repair of a perforated mycotic aneurysm of the right subclavian artery (RSA) in combination with a paravertebral and posterior mediastinal abscess. After resection of the aneurysm and after radical local debridement, orthotopic repair was performed with a self-made pericardial tube graft from the brachiocephalic bifurcation to the thoracic outlet. The paravertebral and posterior mediastinal abscess was drained. The postoperative course was uneventful. Using a self-made readily available pericardial neo-tube enlarges the armamentarium of handling complex infective surgical scenarios and presents a smart alternative to alloplastic vascular reconstruction.
\end{abstract}

\section{Introduction}

Infective systemic complications in patients under continuing immunosuppressive therapy are common, but mycotic arterial aneurysms are very rare. ${ }^{1}$ Vascular reconstruction is usually performed with alloplastic material according to the site and size of the lesion. In native and prosthetic vascular infection after aortic surgery and intervention, self-made pericardial tube-graft have proven to be an attractive treatment option to overcome the issue of reinfection of alloplastic vascular material. ${ }^{2}$ However, there are no reports using this strategy at the level of the supra-aortic branches.

\section{Case Report}

A 63-year-old female with a history of kidney transplantation was admitted to a referring hospital due to gastroenteritis symptoms, dehydration, urinary tract infection, and fever. Initial X-ray showed a right superior mediastinal mass, which was interpreted as a pulmonary or mediastinal abscess, respectively. The patient had been initially treated with wide spectrum antibiotic therapy for a week, but the symptoms and fever persisted. Further diagnostics now using a computed tomography (CT) angiography showed a $3.5 \times 2.9 \mathrm{~cm}$ mycotic aneurysm of the intrathoracic right subclavian artery (RSA) beginning $2 \mathrm{~cm}$ distal to the offspring from the brachiocephalic trunk ending at the level of the thoracic outlet uncompressing the offspring of the right vertebral artery and the right internal mammary artery (-Fig. 1). In addition, paravertebral and posterior mediastinal abscesses were seen. Furthermore, venous obstruction due to compression of the venous confluence was observed. Consecutively, the patient was transferred to our hospital, and emergency surgery was performed.

Initially, the extrathoracic RSA as well as the right common carotid (RCCA) artery was circumferentially dissected and encircled with silastic tapes for distal bleeding control. Then, a median sternotomy was performed, and the central brachiocephalic trunk was secured. Afterward, the RSA was dissected centrally and clamped after systemic heparinization with received

November 13, 2017

accepted after revision

December 19, 2017
DOI https://doi.org/

10.1055/s-0038-1636940. ISSN 2194-7635.
๑) 2018 Georg Thieme Verlag KG
Stuttgart · New York

License terms

(®) $\Theta \circledast$ 


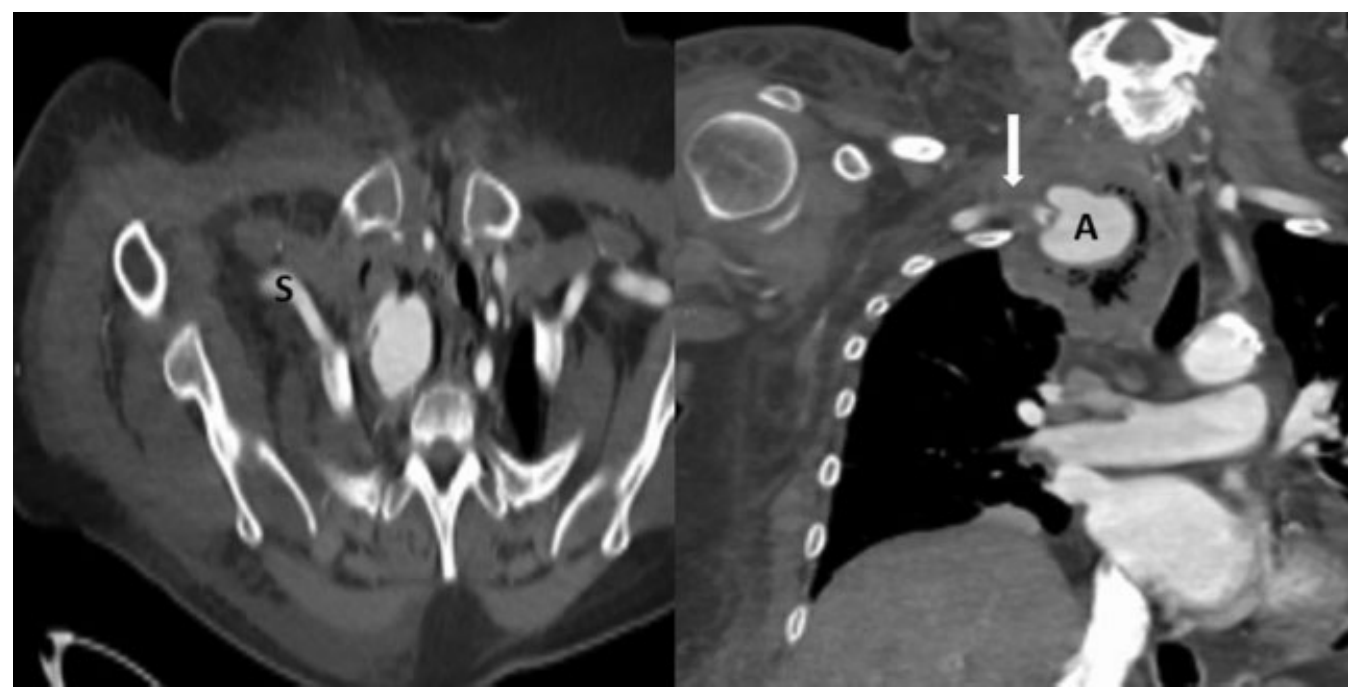

Fig. 1 Pre-operative CT angiography showing a partially thrombosed aneurysm (A) of the right subclavian artery (S) with air lucencies and severe inflammatory changes. Also, a thrombus (arrow) is seen in the subclavian artery, directly distal to the aneurysm. Edematous subcutaneous tissue of the right chest wall, right axial, and right neck root as well as right shoulder muscles. CT, computed tomography.

$100 \mathrm{IU} / \mathrm{kg}$ bodyweight. In addition, the extrathoracic RSA and the RCCA were clamped. Then, the abscess formation was opened. Swabs were taken. The posterior wall of the intrathoracic RSA was completely destructed by the infective process, and discontinuity at the level of the right vertebral artery offspring could be observed (-Fig. 2). Remaining back bleeding from the RSA was controlled via a Fogarty catheter. After radical local debridement, orthotopic repair was performed with a self-made pericardial tube graft from the brachiocephalic bifurcation to the thoracic outlet. For this purpose, a $14 \times 8 \mathrm{~cm}$ bovine pericardial patch (LeMaitre XenoSure Biologic Patch) was sized down to $8 \times 4.8 \mathrm{~cm}$ and was sewn to form a tubular graft with a diameter of $1.5 \mathrm{~cm}$ and a length of $8 \mathrm{~cm}$. The continuing suture row was sewn with 4-0 Prolene (polypropylene) suture (Ethicon, Summerville, New Jersey, United States), and a knot was made at $4 \mathrm{~cm}$ to allow the use of a shorter segment if necessary. ${ }^{2}$ The vertebral artery was not involved and was maintained well.
The paravertebral and posterior mediastinal abscess was drained via opening of the right pleural cavity. Afterward, the wound was closed in layers. The postoperative course was uneventful. Microbiology revealed Escherichia coli as underlying bacterium. An intravenous targeted antibiotic had been continued for 2 weeks. Completion CT scan showed regular arterial perfusion of the right upper extremity and a regular situs without any remaining signs of remaining or recurring infection (-Fig. 3 ). The regular control after 1 and 5 months with Duplex sonography showed good patency of the graft.

\section{Discussion}

Mycotic arterial aneurysms in patients either under chronic immunosuppression or in an immunocompromised state due to infection, such as human immunodeficiency virus (HIV) are very rare. ${ }^{1,3}$ However, mycotic aneurysms of the

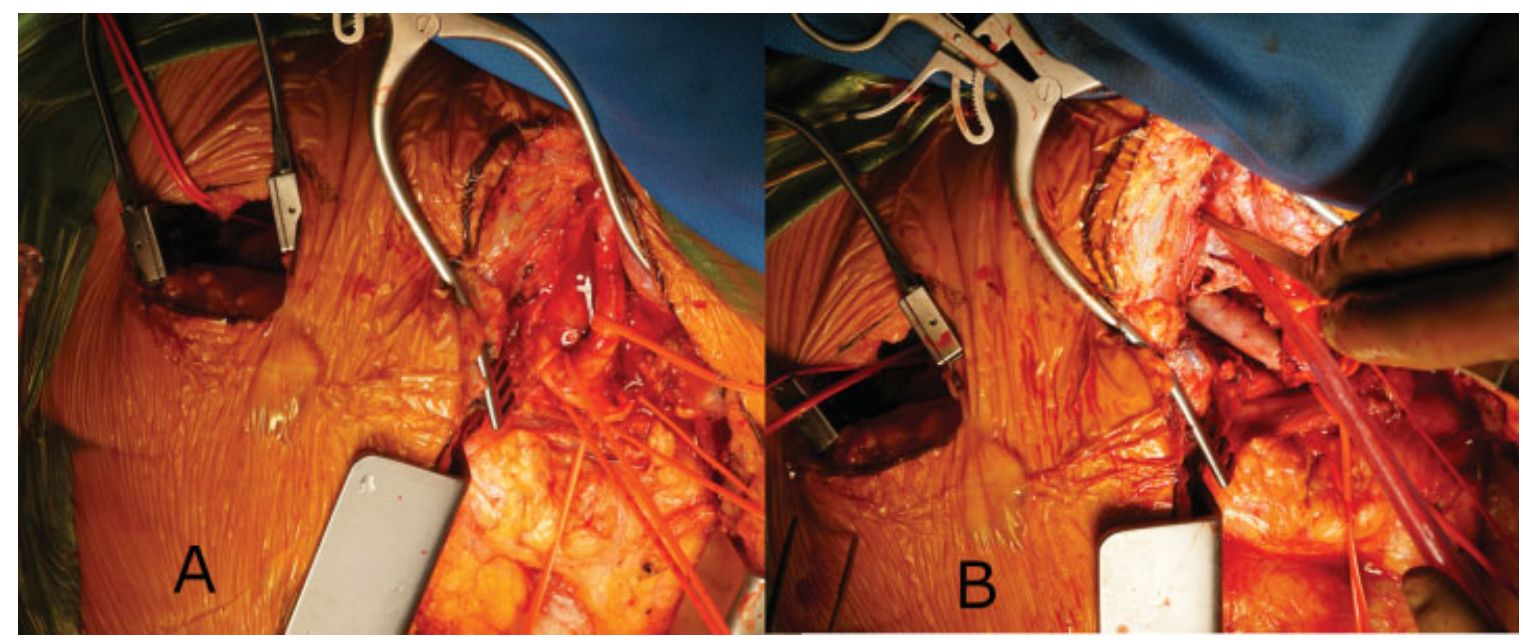

Fig. 2 Intraoperative situs before opening of the abscess formation (A). Intraoperative situs after neopericardial tube graft insertion (B). 


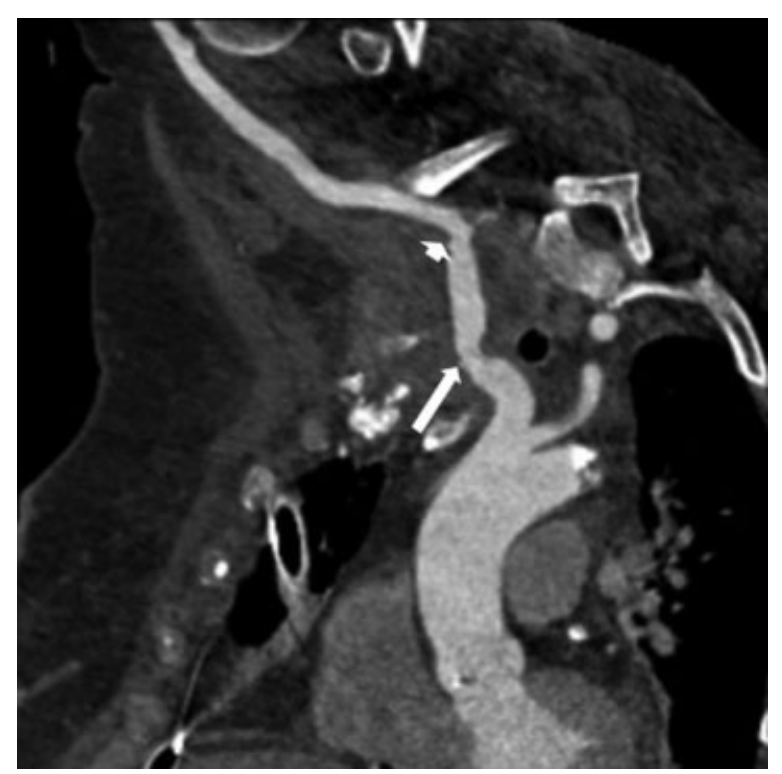

Fig. 3 Reconstructed postoperative CT angiography images showing the reconstructed right subclavian artery with regression of the air lucencies and patent subclavian artery with good distal run off. Arrow: proximal anastomosis, Arrow head: distal anastomosis. CT, computed tomography.

subclavian arteries in both groups at risk have been reported. In a group of patients with HIV infection, a large series reporting 40 arterial aneurysms, merely 2 were located at the level of the subclavian arteries. ${ }^{3}$

Symptoms are usually related to size, location, and affection of adjacent structures. Intrathoracic subclavian artery aneurysms may cause dysphagia, upper limb edema, and Horner's syndrome, whereas extrathoracic subclavian artery aneurysms may compress the cervical plexus causing dysesthesia or even motoric deficits. ${ }^{4}$ However, symptoms are unspecific, and suspicion is needed to establish the diagnosis. In case of suspicion, liberal uses of imaging modalities, such as $\mathrm{CT}$ angiography, are recommended.

Several treatment options, besides germ-oriented antibiotic therapy are available. Endovascular therapy has been used. However, this approach requires insertion of alloplastic vascular material and may be seen as a bridge to definitive therapy as the likelihood of additional infection of the implanted covered stent is to be foreseen. ${ }^{5}$ Saphenous vein graft interposition is an attractive option. However, this technique requires a similar diameter between the native artery and the venous interposition, which is not always given. ${ }^{6}$ Simple ligation of the native vessel has been used. However, collateral flow should be sufficient to provide adequate flow to the respective upper extremity. Finally, claudication on exertion or even limb ischemia should be considered as an adverse effect while using this approach. ${ }^{4}$

Using alloplastical material for classical surgical reconstruction has been successfully reported. However, the remaining risk of recurring infection remains, and antibiotic therapy has to be extensively continued.

Self-made pericardial tube grafts are readily available, can be tailored to the individual size needed, and have been reported to be a very durable solution in case of native and prosthetic aortic infection. ${ }^{2}$ We sought to transfer this concept in this emergency scenario anticipating similar favorable outcome in the supra-aortic position. In addition, site-harvesting complications, in particular, in patients under immunosuppression, can be avoided.

In brief, using a self-made readily available pericardial neo-tube enlarges the armamentarium of handling complex infective surgical scenarios and presents a smart alternative to alloplastic vascular reconstruction.

\section{Conflict of Interest \\ None.}

\section{References}

1 Rabitsch W, Brugger SA, Trubel W, Keil F, Greinix HT, Kalhs P. Streptococcus pneumoniae mycotic aortic aneurysm after allogeneic bone marrow transplantation. Transplantation 2002;74 (07):1048-1050

2 Czerny M, von Allmen R, Opfermann P, et al. Self-made pericardial tube graft: a new surgical concept for treatment of graft infections after thoracic and abdominal aortic procedures. Ann Thorac Surg 2011;92(05):1657-1662

3 Wong JM, Shermak MA, Tihan T, Jones CE. A subclavian artery aneurysm in a patient with HIV infection: a case report. J Vasc Surg 2002;35(05):1006-1009

4 Saliou C, Badia P, Duteille F, D’Attellis N, Ricco JB, Barbier J. Mycotic aneurysm of the left subclavian artery presented with hemoptysis in an immunosuppressed man: case report and review of literature. J Vasc Surg 1995;21(04):697-702

5 Ohkoshi Y, Ninomiya H, Mukai HY, et al. Pseudoaneurysm of the subclavian artery due to Xanthomonas pneumonia in a patient with acute myeloid leukemia: its rupture treated by transcatheter coil embolization. Intern Med 1999;38(08):671-674

6 Marks C, Kuskov S. Pattern of arterial aneurysms in acquired immunodeficiency disease. World J Surg 1995;19(01):127-132 